\title{
Failure to recognise tardive dyskinesia in the long stay population
}

\author{
Robertson MacPherson, Registrar in Psychiatry; and Rachel Collis, Registrar in \\ Psychiatry, Northern General Hospital, Sheffield S5 7AU
}

Tardive dyskinesia (TD) is an involuntary movement disorder associated with long term neuroleptic exposure. Gerlach \& Casey (1988) reported a $15 \%$ prevalence rate of TD among patients on neuroleptics, the rate increasing to $54 \%$ in patients over 60 years old.

Early diagnosis of TD is important because it is estimated (Marsden, 1985) that if neuroleptic medication can be stopped, $60 \%$ of TD cases will resolve within five years. Continuing medication has been shown to cause a worsening of TD in a proportion of cases. To date no effective long term treatment for established TD has been demonstrated.

In this study we screened long stay psychiatric patients for signs of movement disorder. We sought to establish the level of awareness of the presence of TD among the medical and nursing staff caring for the patients. We also wanted to examine whether any relationship existed between the clinical features of movement disorder and staff awareness.

\section{The study}

Patients on eight long stay wards of Middlewood Hospital, Sheffield, were screened for signs of movement disorder using the Abnormal Involuntary Movement Scale (AIMS, Guy, 1976). The following criteria were used to diagnose TD: total AIMS score greater than 5 , global severity rating 1 or more, movement disorder at least moderately severe in 1 or more body areas. Patients with movement disorder were questioned in detail about their awareness of it, and any consequent distress or disability. Patients' responses to these questions were recorded verbatim, and they were considered to be unaware only if there was true denial, or a total lack of awareness was apparent.

Senior nursing staff from each ward completed a brief questionnaire for all patients on the ward. The nurses were asked to report whether they thought each patient had TD, and for those patients considered to have TD, to rate its severity and the degree of distress and disability caused.

Medical case-notes were examined for any mention of movement disorder or related conditions, and the presence of any diagnosis other than TD, which could be an alternative explanation for movement disorder.

\section{Findings}

Of 113 patients screened, $43(38.1 \%)$ satisfied the criteria for TD. Four patients refused to participate in the assessments of self perception of movement disorder and have therefore been excluded from this part of the analysis. They have, however, been included in the analysis of the levels of staff awareness of movement disorder.

Of the 39 consenting patients with TD, 13 were considered to be aware of movement disorder. The assessors' ratings of distress caused by movement disorder in the aware group were as follows: no distress, 4 patients; moderate distress, 7 patients; severe distress, 2 patients. None of the unaware patients were considered to be distressed by their movement disorder. Five patients reported that movement disorder interfered with activities.

Nursing staff were aware of TD in 18 of the 43 affected patients. The mean total AIMS score of these 18 patients was higher than the mean score of the patients whose movement disorder was not 'picked up' by the nurses (mean score 19.1 (s.d. 6.8) v. 14.0 (s.d. 5.1), Mann Whitney $U$ test $P<0.02$ ). Nursing staff awareness of TD was not found to be related to the site of movement disorder, the nurses picking up a similar proportion of patients with predominantly orofacial dyskinesia, and peripheral dyskinesia. Neither was nursing staff awareness related to patients' awareness of movement disorder, nor to the presence of spontaneous complaints by the patients. One patient who was considered by nursing staff to have TD was found on examination to have antalgic gait secondary to osteoarthritis.

Medical casenotes recorded a specific diagnosis of TD in six cases, but in eight further cases abnormal involuntary movements of some sort were recorded. In only one instance was a management plan made as a result of the diagnosis of TD. Interestingly, one patient with movement disorder had multiple investigations including a CT scan to exclude Huntington's chorea, but there was no mention of TD in the differential diagnosis. All six of the patients positively 
diagnosed in the casenotes denied awareness of movement disorder. Nursing staff awareness coincided with medical diagnosis in half the cases (three out of six patients).

\section{Comment}

Patients on long stay wards are an often neglected group, and the findings of this study give further cause for concern. A high proportion (38.1\%) of the population studied had a well recognised side effect of long term neuroleptic medication, TD, but in only six out of 43 cases $(14 \%)$ was the diagnosis recorded in the medical casenotes. Furthermore, in only one case out of $\mathbf{4 3}$ was an appropriate management plan established as a result of the diagnosis, despite widely documented evidence of the long term benefits of stopping or reducing neuroleptic medication when this side effect has occurred.

We have attempted to find a relationship between the clinical features of TD and the low levels of awareness among medical and nursing staff of the disorder. In this population we have demonstrated that nursing staff are more likely to 'pick up' cases which are severe than they are to be aware of those patients with TD who complain of the disorder, or who have been diagnosed by medical colleagues. The low level of diagnosis in the medical casenotes makes analysis of the determinants of medical diagnosis difficult. It is, however, suggested that improved sharing of information between medical and nursing staff could greatly improve the level of medical diagnosis in this population, and that as the main 'carers', nursing staff may be well placed to help in establishing both the presence of TD and in implementing a management plan regarding further treatment.

We have demonstrated low levels of awareness of movement disorder in the population of patients with TD, and even lower levels of complaints expressed by the patents. We feel that this emphasises the need for vigilance on the part of psychiatrists prescribing to these patients, and demonstrates the complexity of the issues regarding patients' ability to give informed consent for neuroleptic treatment. As
DeVeaugh-Geiss (1979) said: "Can a person whose thinking itself is modified by a treatment properly give consent to continuing that treatment, especially if it could reasonably be expected that discontinuing the treatment would result in refusal to consent? Is a person who has been disabled by treatment able to continue treatment and accept exposure to the risk when he denies the disability and, implicitly, the risk involved?

In the United States, public concern regarding TD has been rising, and the American Psychiatric Association Task Force report (Baldessarini et al, 1979) recommended the implementation of an informed consent procedure, prior to embarking on long term prophylactic neuroleptic therapy. The issue of risk/benefit analysis regarding the long term prescription of neuroleptic drugs is one which regularly faces all general psychiatrists, and the general use of a structured consent procedure would help to bring this public health issue to wider attention among prescribing psychiatrists.

\section{Acknowledgement}

The authors would like to thank Dr Malcolm Peet for his help and advice in the preparation of this paper.

\section{References}

Baldessarin, R. J., Cole, J. D., Davis, J. M. et al (1979) Report of the American Psychiatric Association Task Force on Late Neurological Effects of Antipsychotic Drugs. Washington, DC: American Psychiatric Association, p. 18.

DEVeaugh-Geiss, J. (1979) Informed consent for neuroleptic therapy. American Journal of Psychiatry, 136, 959-962.

Gerlach, J. \& CaseY, D. E. (1988) Tardive dyskinesia. Acta Psychiatrica Scandinavica, 77, 369-378.

GUY, W. (1976) ECDEU Assessment Manual for Psychopharmacology. Washington, DC: US Department of Health Education and Welfare.

MARSDEN, C. D. (1985) Is tardive dyskinesia a unique disorder? In: Dyskinesia - research and treatment (eds D. E. Casey, T. Chase, A. V. Christensen and J. Gerlach). Berlin: Springer. 